\title{
Effect of gender, gonadectomy and oestradiol-17ß on growth in lambs under grazing conditions
}

\author{
O. MAHGOUB ${ }^{1}$, G. K. BARRELL ${ }^{2 *}$ AND A. R. SYKES ${ }^{2}$ \\ ${ }^{1}$ Department of Animal and Veterinary Science, Faculty of Agriculture, Sultan Qaboos University, \\ P.O. Box 34, Al Khod 123, Sultanate of Oman \\ ${ }^{2}$ Animal and Food Sciences Division, P.O. Box 84, Lincoln University, Canterbury, New Zealand
}

(Revised MS received 8 May 2001)

\begin{abstract}
SUMMARY
To identify separate effects of gender, castration and exogenous oestrogen on growth, castrated lambs of both sexes and entire male lambs $(n=8)$ were implanted subcutaneously with three sizes of oestradiol-17 $\beta$ implants, or not implanted, and grazed on ryegrass and white clover pasture for 180 days. A group of non-implanted entire female lambs $(n=8)$ was run together with the others. Nonimplanted entire male lambs grew faster, had heavier heads, less internal, non-carcass fat and more protein and less fat and water in the carcass than non-implanted entire females. In addition, they had higher 12th vertebral spine, thicker tibia, and heavier and larger humerus than entire female lambs. Castration of male lambs reduced live-weight gain, weight of head and content of protein in the carcass whereas it increased carcass fat content. In addition, it caused lengthening of cannon bones and reduced height of 12 th vertebral spine and length of tibia. In females, gonadectomy increased height of 12 th vertebral spine and diameter to length ratio of the radius. Oestradiol treatment increased live-weight gain, reduced total internal and carcass fat, and increased water and protein content of the carcass in gonadectomized animals of either sex, and increased weight of carcass and head in spayed ewe lambs. Oestradiol treatment inhibited longitudinal growth of cannon bones and stimulated that of vertebral column and ribs, but had little effect on the dimensions of limb bones apart from increasing their diameter. Oestradiol treatment had no effect on muscle length but increased muscle girth and weight, except for m. splenius in ram lambs where muscle weight was reduced. Effects of oestradiol on skeletal measurements in most cases were linearly related to dose of oestradiol. It was concluded that the variable effects of sex steroids on the skeleton were related to the differential pattern of skeletal maturation. In early maturing bones acceleration of the growth process by an exogenous sex steroid caused elongation to cease prematurely, whereas in late-maturing bones the acceleration effect on elongation did not result in premature cessation. This observation may explain the often contradictory reports in the literature on the effects of sex steroids on linear growth of bone.
\end{abstract}

\section{INTRODUCTION}

Oestrogens have been used widely as growth promoters in farm animals (Galbraith \& Topps 1981; Hancock et al. 1991), yet they appear to inhibit skeletal growth in laboratory animals and man (Silberberg \& Silberberg 1972; Short 1980). For instance oestrogen treatment stimulated linear growth of vertebral column and cannon bones (Wilkinson et al. 1955) and growth at the distal end of the femur (Shroder \& Hansard 1959) in sheep, whereas it inhibited linear growth of limb bones, vertebrae and

* To whom all correspondence should be addressed. Email: barrell@lincoln.ac.nz ribs in rats (Zondek 1936). These contradictory effects of oestrogens have been attributed to variation between species, in ranges of doses, ages of animals, duration of treatment or chemical identity of the hormone molecule used (Silberberg \& Silberberg 1972; Spencer 1985). Dose effects were explained by the hypothesis that small doses of these hormones stimulate linear growth of bone whereas large doses inhibit it (Suzuki 1958; Short 1980). Experiments which have studied effects of lack of sex hormones by use of castration have also shown contradictory findings. Castration of male, and to a lesser extent of female, ruminants lengthened limb bones, particularly distal limb bones, resulting in animals which were taller than entires (Brannang 1971 $a, b$ ). On the other 
hand, castration shortened the vertebral column resulting in animals shorter in body length than entires (Tandler \& Keller 1910-1911; Brannang $1971 a, b)$.

Skeletons of animals mature in a differential manner (Hammond 1932; Wallace 1948; Davies 1979; Davies et al. 1984) with the skull and cannon bones maturing earlier in life and the vertebral column later. The extent to which activity of epiphyseal plates can be affected by oestrogens at any particular stage of growth may vary for different bones in the skeleton. The present study examined the effects of endogenous sex hormones and an exogenous oestrogen, oestradiol, on skeletal growth of sheep in relation to the differential pattern of skeletal maturation during their first 8 months of postnatal growth.

\section{MATERIALS AND METHODS}

\section{Experimental}

One hundred and four Dorset Down X Coopworth lambs comprising 64 males and 40 females aged 8 to 10 weeks (mean live weight $=20.4 \mathrm{~kg}$ ) were used in the experiment. Thirty-two of both sexes were gonadectomized at $8-10$ weeks and were randomly allocated, within gender, to 13 groups $(n=8)$ (Table $1)$. Four groups (one of each sex class) were nonimplanted controls ( 0 ) and the other nine groups (three each of wether, ram and spayed ewes) were treated with low (L, 2 implants), medium (M, 1 implant) and high ( $\mathrm{H}, 1$ implant) dose oestrogen implants (Table 1) one week after the last gonadectomy operation. Animals were grazed throughout the experimental period of 180 days on pasture consisting predominantly of perennial ryegrass (Lolium perenne L.) and white clover (Trifolium repens L.). The implants were formed of silicone rubber containing oestradiol-17 $\beta$ moulded in three sizes; small (L) (surface area, SA, $75 \mathrm{~mm}^{2}$ ), medium (M) (SA $\left.603 \mathrm{~mm}^{2}\right)$ and large $(\mathrm{H})\left(\mathrm{SA} 1574 \mathrm{~mm}^{2}\right)$ which contained 3, 22 and $52 \mathrm{mg}$ oestradiol, respectively. Animals with the $2 \mathrm{~L}$ dose implants were re-implanted

Table 1. Allocation of lambs to sex, gonadectomy and oestrogen treatment groups $(0=$ control, $L=$ low dose (6 $\mathrm{mg}), M=$ medium dose $(22 \mathrm{mg})$ and $H=$ high dose $(52 \mathrm{mg})$ oestradiol implants)

\begin{tabular}{lrrrrr}
\hline \hline & \multicolumn{4}{c}{ Oestrogen dose level } \\
\cline { 2 - 5 } Sex group & 0 & $\mathrm{~L}$ & $\mathrm{M}$ & $\mathrm{H}$ & Total \\
\hline Wethers & 8 & 8 & 8 & 8 & 32 \\
Entire rams & 8 & 8 & 8 & 8 & 32 \\
Spayed ewes & 8 & 8 & 8 & 8 & 32 \\
Entire ewes & 8 & - & - & - & 8 \\
Total & 32 & 24 & 24 & 24 & 104 \\
\hline \hline
\end{tabular}

with another 2 implants 41 days later to overcome the possibility of exhaustion of hormone from the implants. Blood samples $(10 \mathrm{ml})$ were collected on the day before implantation and at 1 and 175 days after implantation into heparinized glass tubes, centrifuged and the plasma stored at $-20^{\circ} \mathrm{C}$. Plasma oestradiol concentration was measured by radioimmunoassay (Peterson et al. 1975). All procedures involving the use of animals were approved by the Lincoln University Animal Ethics Committee.

\section{Measurements}

Lambs were weighed weekly and length of right and left fore cannon bones was measured every 3 weeks to the nearest $\mathrm{mm}$ using vernier calipers. At the end of 180 days (at approximately 35 weeks of age, mean live weight 39 to $45 \mathrm{~kg}$ ), the lambs were slaughtered and beheaded by severing the atlanto-occipital joint, teat lengths were measured and the warm carcasses, heads, testes and uteri were weighed then stored at $-20^{\circ} \mathrm{C}$. The carcass was split along the midline using a band saw. The following measurements were taken from the thawed left half-carcasses before dissection; soft tissue depth over the 11 th rib at $11 \mathrm{~cm}$ from midline of backbone (GR), A (length) and B (width of eye muscle, $m$. longissimus thoracis et lumborum, LD) and C (fat thickness over the 12th rib). A, B and C measurements were carried out according to the methods of Pálsson (1939). Whole right half-carcasses were minced and subsampled for chemical analysis. Subsamples were weighed, freeze-dried for 4 days, reweighed (to determine water content), ground and analysed for protein and fat content following the procedures of AOAC (1984). The frozen left halfcarcasses were thawed and the cervical, thoracic, lumbar and sacral regions of the vertebral column were identified according to Getty (1975). Each region was measured in length and the number of vertebrae was recorded. The 1st and 12th ribs and their corresponding vertebral spines were exposed and each length of rib and height of spine was measured. The radius and ulna, humerus, femur and tibia were dissected out and measured in length and the humerus was weighed. For each of these bones maximum diameter at mid-point was measured to the nearest $\mathrm{mm}$ using vernier calipers. Femur bone was sawn transversely at mid-point and maximum thickness of cortex at this site (FMCT) was measured using the same calipers. The 12th rib was dissected out, separated from adhering tissue, dried in an oven at $110^{\circ} \mathrm{C}$ for $24 \mathrm{~h}$, then incinerated in a muffle furnace at $550^{\circ} \mathrm{C}$ for $16 \mathrm{~h}$. Ash proportion (ash weight divided by dry matter weight, $\mathrm{g} / \mathrm{kg}$ ) and ash to organic matter ratio $(\mathrm{A}: \mathrm{R})$, calculated as ash weight divided by (dry matter weight minus ash weight), were determined. Muscles, $m$. biceps brachii (BIC) and $m$. extensor carpi radialis (ECR), were exposed and 
measured for length (from origin to insertion) and maximum girth (at thickest point) in situ then dissected out and weighed. M. splenius was also dissected out and weighed.

\section{Statistical methods}

Experimental data were analysed utilizing orthogonal polynomial contrasts (Alvey et al. 1980) for differences between control and treated animals. The same analysis was used to study the following contrasts; entire $v$. gonadectomized; castrated males $v$. spayed females; linear and quadratic dose responses within wether, ram and spayed ewe lambs; sex effect (rams $v$. entire ewes). The logarithmic form of the Gompertz equation:

$$
\log \mathrm{e} W=\log \mathrm{e}^{-\mathrm{be}^{-\mathrm{kt}}}
$$

(where $\mathrm{W}$ is size at time $\mathrm{t}$ and $\mathrm{A}$ its ultimate value, $\mathrm{k}$ is the rate constant and $\mathrm{b}$ is time zero (Richards 1959)) was fitted to adjusted mean cannon bone lengths using optimization procedures of the Genstat statistical package (Alvey et al. 1980). The equation coefficients and their standard errors plus the residual standard deviation were used to compare elongation of cannon bones between different experimental groups during the experimental period. Other bone data were adjusted to mean initial radius length by analysis of covariance. Data from wether and spayed ewe lambs were pooled and analysed by use of orthogonal polynomial contrasts for differences between control and oestradiol-treated animals and for linear and quadratic dose responses.

\section{RESULTS}

The general trend was for faster live-weight gain in male lambs and for reduced fatness with oestradiol treatment.

\section{Effectiveness of oestradiol implants}

Dose of oestradiol (calculated as mean weight loss from implants) was $2 \cdot 6,6 \cdot 0$ and $16 \cdot 4 \mathrm{mg}$ (i.e. $14 \cdot 4,33 \cdot 3$ and $91 \cdot 1 \mu \mathrm{g} /$ day, assuming constant rate of release) for low, medium and high dose groups respectively (Table 2). Oestradiol concentration in plasma of implanted wethers was elevated immediately after implantation but was near to pre-implantation values at the end of the experiment (Table 3 ).

\section{Effects of sex-entire males $v$. entire females}

Entire ram lambs tended to have a higher rate of liveweight gain than entire ewe lambs (coefficient $\mathrm{k}$, Table 4). Although the rate of elongation of cannon bones was not different to that of entire female lambs (Tables 5 and 7), the ram lambs tended to have a longer vertebral column, thicker limb bones with greater diameter:length ratios and generally heavier and larger bones (Table 5). The only significant differences, however, were longer 12th vertebral spine, thicker tibia bone and heavier humerus (Tables 5 and 6). Ram lambs had significantly thicker BIC and ECR muscles and heavier BIC and splenius muscles than ewe lambs (Tables 8 and 9). In addition, they had heavier head and greater protein and water content than entire ewe lambs, whereas the latter animals had greater carcass and non-carcass fat content (Tables 10 and 11).

\section{Effects of sex-castrated males versus spayed females}

Control wether lambs tended to have slower liveweight gain (coefficient $\mathrm{k}$, Table 4) and had less noncarcass fat than control spayed female lambs (Tables

Table 3. Mean plasma oestradiol-17 $\beta$ concentration $(\mathrm{pg} / \mathrm{ml}) \pm$ S.E.M. of non-implanted lambs (control) and wether lambs implanted with silicone rubber implants containing oestradiol- $17 \beta$ recorded at 3 stages of the trial $(\mathrm{n}=8)$

\begin{tabular}{llcc}
\hline \hline \multirow{2}{*}{ Group } & \multicolumn{3}{c}{ Day of experiment } \\
\cline { 2 - 4 } & Pre-implantation & 1 & 175 \\
\hline Control wethers & $30^{*}$ & - & - \\
Control rams & $20 \pm 8$ & - & $24 \pm 4$ \\
Control spayed ewes & $43^{*}$ & - & - \\
Control entire ewes & $13 \pm 3$ & - & $26 \pm 7$ \\
Low dose wethers & - & $136 \pm 44$ & $29 \pm 8$ \\
Medium dose wethers & $43^{*}$ & $102 \pm 47$ & $42 \pm 12$ \\
High dose wethers & $30^{*}$ & $167 \pm 60$ & $31 \pm 13$ \\
\hline \hline
\end{tabular}

* Oestradiol concentration was obtained from pooled samples, collected at least 1 week after gonadectomy in the case of wethers and spayed ewes.

Table 2. Loss of weight $(\mathrm{mg})$ from small $(L)$, medium $(M)$ and large $(H)$ silicone rubber implants containing oestradiol-17 $\beta$, after 180 days subcutaneous implantation in lambs $(\mathrm{n}=8)$

\begin{tabular}{lccc}
\hline \hline & L (initial content & M (initial content & H (initial content \\
Group & 6 mg oestradiol) & $22 \mathrm{mg}$ oestradiol) & $52 \mathrm{mg}$ oestradiol) \\
\hline Entire rams & $2 \cdot 5$ & $6 \cdot 0$ & $22 \cdot 7$ \\
Wethers & $2 \cdot 6$ & $6 \cdot 1$ & $11 \cdot 7$ \\
Spayed ewes & $2 \cdot 8$ & $6 \cdot 0$ & $14 \cdot 7$ \\
\hline \hline
\end{tabular}


Table 4. Coefficients $A, b$ and $k$ (and their standard errors, $S_{a}, S_{b}$ and $S_{k}$ ) from Gompertz equations fitted to mean live weights (adjusted to initial live weight) of control and treated wether, ram, spayed ewe and entire ewe lambs implanted with silicone rubber implants containing low, medium or high doses of oestradiol-17 $\beta(\mathrm{n}=8)(r s d=$ residual standard deviation)

\begin{tabular}{lccccccc}
\hline \hline Group & $\mathrm{A}($ antilog) & $\left(\mathrm{S}_{\mathrm{A}}\right)$ & $\mathrm{B}$ & $\left(\mathrm{S}_{\mathrm{b}}\right)$ & $\mathrm{k}$ & $\left(\mathrm{S}_{\mathrm{k}}\right)$ & rsd \\
\hline Control wethers & $36 \cdot 21$ & $0 \cdot 0237$ & $0 \cdot 1566$ & $0 \cdot 0185$ & $0 \cdot 0225$ & $0 \cdot 0095$ & $0 \cdot 0236$ \\
Low dose wethers & $40 \cdot 10$ & $0 \cdot 0464$ & $0 \cdot 1756$ & $0 \cdot 0246$ & $0 \cdot 0154$ & $0 \cdot 0080$ & $0 \cdot 0274$ \\
Medium dose wethers & $40 \cdot 36$ & $0 \cdot 0439$ & $0 \cdot 1823$ & $0 \cdot 0234$ & $0 \cdot 0123$ & $0 \cdot 0049$ & $0 \cdot 0204$ \\
High dose wethers & $41 \cdot 01$ & $0 \cdot 0549$ & $0 \cdot 1768$ & $0 \cdot 0281$ & $0 \cdot 0140$ & $0 \cdot 0079$ & $0 \cdot 0282$ \\
Control rams & $38 \cdot 52$ & $0 \cdot 0332$ & $0 \cdot 1692$ & $0 \cdot 0222$ & $0 \cdot 0201$ & $0 \cdot 0099$ & $0 \cdot 0284$ \\
Low dose rams & $37 \cdot 93$ & $0 \cdot 0327$ & $0 \cdot 1611$ & $0 \cdot 0212$ & $0 \cdot 0193$ & $0 \cdot 0095$ & $0 \cdot 0270$ \\
Medium dose rams & $40 \cdot 48$ & $0 \cdot 0349$ & $0 \cdot 1809$ & $0 \cdot 0201$ & $0 \cdot 0160$ & $0 \cdot 0062$ & $0 \cdot 0236$ \\
High dose rams & $40 \cdot 00$ & $0 \cdot 0296$ & $0 \cdot 1772$ & $0 \cdot 0200$ & $0 \cdot 0192$ & $0 \cdot 0076$ & $0 \cdot 0256$ \\
Control spayed ewes & $37 \cdot 48$ & $0 \cdot 0275$ & $0 \cdot 1609$ & $0 \cdot 0191$ & $0 \cdot 0200$ & $0 \cdot 0085$ & $0 \cdot 0243$ \\
Low dose spayed ewes & $40 \cdot 21$ & $0 \cdot 0360$ & $0 \cdot 1805$ & $0 \cdot 0217$ & $0 \cdot 0169$ & $0 \cdot 0071$ & $0 \cdot 0264$ \\
Medium dose spayed ewes & $40 \cdot 23$ & $0 \cdot 0373$ & $0 \cdot 1743$ & $0 \cdot 0221$ & $0 \cdot 0171$ & $0 \cdot 0078$ & $0 \cdot 0270$ \\
High dose spayed ewes & $42 \cdot 82$ & $0 \cdot 0356$ & $0 \cdot 1935$ & $0 \cdot 0208$ & $0 \cdot 0164$ & $0 \cdot 0016$ & $0 \cdot 0252$ \\
Control entire ewes & $37 \cdot 85$ & $0 \cdot 0235$ & $0 \cdot 1682$ & $0 \cdot 0159$ & $0 \cdot 0186$ & $0 \cdot 0060$ & $0 \cdot 0181$ \\
\hline \hline
\end{tabular}

10 and 11). Also, the wether lambs had longer vertebral column, longer 1 st vertebral spine and larger and heavier humerus bone, but shorter 1 st rib (Tables 5 and 6). In the case of muscles, wether lambs had longer, thinner and lighter ECR but heavier splenius muscles than spayed females (Tables 8 and 9).

\section{Effects of gonadectomy - general}

In male lambs, castration reduced final live weight (coefficient A, Table 4 and Table 10), weight of the head, A measurement of the LD muscle and protein and water content in the carcass, whereas it increased $\mathrm{C}$ measurement of fat depth and fat content (Tables 10 and 11). Ovariectomy in females had very little effect on live-weight or on most body components except the uterus (Tables 10 and 11). Spayed ewe lambs had shorter teats than entire ewes and their uteri were one third of the weight of those of entire ewes (Tables 10 and 11).

\section{Effects of gonadectomy - male lambs}

In male lambs castration increased growth in length of cannon bones but reduced length of the 12th vertebral spine and length of tibia (Tables 5, 6 and 7). Castration significantly reduced the girth of BIC and ECR muscles and the weight of ECR and splenius muscles (Tables 8 and 9).

\section{Effects of gonadectomy - female lambs}

Ovariectomy increased elongation of cannon bones and increased length of the vertebral column, 12th vertebral spine and radius diameter:length ratio (Tables 5,6 and 7) but did not affect any of the muscle measurements (Tables 8 and 9).

\section{Effects of oestradiol implants - general}

Oestradiol treatment tended to increase final live weight in gonadectomized lambs of both sexes and had minor growth stimulatory effects in entire rams (Tables 10 and 11, coefficient A in Table 4). For many skeletal components there was a positive linear relationship with the dose of the hormone implant although in a few cases, within sex class, there appeared to be a quadratic response (Tables 5 and 6).

\section{Effects of oestradiol implants - gonadectomized lambs}

Oestradiol treatment reduced ultimate length of cannon bones (coefficient A, Table 7). Cannon bones of treated animals gained less in absolute terms during the treatment period and consequently were shorter at the end of the experiment than in control lambs (Tables 5 and 6). Oestradiol-treated wether and spayed ewe lambs tended to have longer vertebral column and 12th rib than their relative controls. However, there was no significant effect of oestradiol on length of medial limb bones, i.e. radius and ulna, humerus, femur and tibia (Tables 5 and 6). Oestradiol treatment tended to increase diameter of limb bones in gonadectomized lambs of both sexes, with the effect being significant in the case of the femur (Tables 5 and 6) and it increased cortical thickness of the femur, ash proportion and ash:organic matter ratio of the rib in wethers (Tables 5 and 6). Treatment with oestradiol did not significantly affect muscle length in gonadectomized lambs but girth and weight of muscles, e.g. BIC muscle, were generally increased (Tables 8 and 9). Because of similar trends in the responses of animals to oestradiol treatment, data from castrated male and spayed female lambs were 
Table 5. Mean length $(\mathrm{mm})$, diameter $(\mathrm{mm})$ and other measurements of some bones adjusted to mean initial radius length $(\mathrm{n}=8)$ and difference between control $(C)$ and treated $(T)$ wether, ram, spayed ewe and entire ewe lambs implanted with low $(L)$, medium $(M)$ or high $(H)$ dose silicone implants containing oestradiol-17 $\beta$

\begin{tabular}{|c|c|c|c|c|c|c|c|c|c|c|c|c|c|c|c|c|c|}
\hline & \multicolumn{4}{|c|}{ Wethers } & \multirow[b]{2}{*}{$\mathrm{C} v . \mathrm{T}$} & \multicolumn{4}{|c|}{ Rams } & \multirow[b]{2}{*}{$\mathrm{C} v . \mathrm{T}$} & \multicolumn{4}{|c|}{ Spayed ewes } & \multirow{2}{*}{\multicolumn{2}{|c|}{$\mathrm{C} v . \mathrm{T} \quad \mathrm{C}$}} & \multirow[b]{2}{*}{ ESE } \\
\hline & $\mathrm{C}$ & $\mathrm{L}$ & M & $\mathrm{H}$ & & $\mathrm{C}$ & $\mathrm{L}$ & M & $\mathrm{H}$ & & $\mathrm{C}$ & $\mathrm{L}$ & M & $\mathrm{H}$ & & & \\
\hline \multicolumn{18}{|l|}{ Bone length } \\
\hline Cannon bone length & 148 & 146 & 146 & 144 & $*$ & 145 & 144 & 145 & 145 & & 148 & 143 & 144 & 145 & $* * *$ & 146 & 1 \\
\hline Gain in cannon bone & 13 & 12 & 11 & 10 & $*$ & 11 & 10 & 10 & 11 & & 13 & 9 & 10 & 10 & $* * *$ & 12 & 1 \\
\hline $\begin{array}{l}\text { Total vertebral } \\
\text { column }\end{array}$ & 814 & 825 & 821 & 837 & & 824 & 820 & 816 & 844 & & 811 & 816 & 834 & 838 & & 807 & 12 \\
\hline 1 st rib & 91 & 94 & 94 & 96 & & 96 & 92 & 95 & 97 & & 97 & 94 & 98 & 95 & & 93 & 3 \\
\hline 1st vertebral spine & 46 & 45 & 44 & 43 & & 46 & 45 & 48 & 48 & & 39 & 40 & 39 & 41 & & 42 & 2 \\
\hline 12 th rib & 178 & 184 & 182 & 190 & & 180 & 181 & 191 & 190 & & 180 & 181 & 194 & 192 & & 186 & 5 \\
\hline 12 th vertebral spine & 21 & 22 & 21 & 23 & & 25 & 23 & 18 & 23 & & 23 & 21 & 23 & 24 & & 20 & 1 \\
\hline Radius & 137 & 137 & 137 & 136 & & 137 & 135 & 137 & 136 & & 136 & 135 & 136 & 136 & & 136 & $1 \cdot 31$ \\
\hline Ulna & 169 & 169 & 170 & 167 & & 170 & 168 & 169 & 167 & & 168 & 170 & 169 & 167 & & 168 & 1.90 \\
\hline Humerus & 121 & 121 & 122 & 122 & & 122 & 122 & 122 & 122 & & 119 & 120 & 122 & 120 & & 121 & $1 \cdot 27$ \\
\hline Femur & 154 & 155 & 154 & 157 & & 157 & 153 & 156 & 156 & & 154 & 153 & 156 & 155 & & 155 & 1.81 \\
\hline Tibia & 177 & 179 & 177 & 180 & & 181 & 178 & 180 & 179 & & 178 & 179 & 180 & 178 & & 179 & $2 \cdot 00$ \\
\hline \multicolumn{18}{|l|}{ Bone diameter } \\
\hline Radius & $16 \cdot 7$ & $17 \cdot 7$ & $17 \cdot 6$ & $17 \cdot 6$ & & $17 \cdot 7$ & $18 \cdot 3$ & $17 \cdot 2$ & $18 \cdot 2$ & & $17 \cdot 3$ & $16 \cdot 9$ & $17 \cdot 6$ & $18 \cdot 1$ & & $16 \cdot 6$ & $0 \cdot 57$ \\
\hline Humerus & $18 \cdot 6$ & $19 \cdot 3$ & $19 \cdot 4$ & $19 \cdot 9$ & & $19 \cdot 2$ & $19 \cdot 6$ & $19 \cdot 1$ & $19 \cdot 5$ & & $18 \cdot 2$ & $18 \cdot 5$ & $19 \cdot 1$ & $19 \cdot 4$ & & $18 \cdot 4$ & 0.59 \\
\hline Femur & $18 \cdot 1$ & $19 \cdot 6$ & $19 \cdot 5$ & $19 \cdot 2$ & $*$ & $19 \cdot 1$ & $19 \cdot 0$ & $18 \cdot 6$ & $19 \cdot 2$ & & $18 \cdot 9$ & $18 \cdot 3$ & $19 \cdot 2$ & $19 \cdot 6$ & $*$ & $18 \cdot 5$ & 0.54 \\
\hline Tibia & $15 \cdot 2$ & $15 \cdot 5$ & $15 \cdot 3$ & $15 \cdot 5$ & & $15 \cdot 7$ & $15 \cdot 8$ & $15 \cdot 5$ & $15 \cdot 5$ & & $15 \cdot 3$ & $14 \cdot 8$ & $15 \cdot 3$ & $15 \cdot 8$ & & $14 \cdot 9$ & $0 \cdot 39$ \\
\hline \multicolumn{18}{|l|}{ Diameter:length ratio } \\
\hline Radius & $0 \cdot 12$ & $0 \cdot 13$ & $0 \cdot 13$ & $0 \cdot 13$ & & $0 \cdot 13$ & $0 \cdot 13$ & $0 \cdot 13$ & $0 \cdot 13$ & & $0 \cdot 13$ & $0 \cdot 12$ & $0 \cdot 13$ & $0 \cdot 13$ & & $0 \cdot 12$ & $0 \cdot 00$ \\
\hline Humerus & $0 \cdot 15$ & $0 \cdot 16$ & $0 \cdot 16$ & $0 \cdot 16$ & & $0 \cdot 16$ & $0 \cdot 16$ & $0 \cdot 16$ & $0 \cdot 16$ & & $0 \cdot 15$ & $0 \cdot 15$ & $0 \cdot 16$ & $0 \cdot 16$ & & $0 \cdot 15$ & $0 \cdot 00$ \\
\hline Femur & $0 \cdot 12$ & $0 \cdot 13$ & $0 \cdot 13$ & $0 \cdot 12$ & & $0 \cdot 12$ & $0 \cdot 12$ & $0 \cdot 12$ & $0 \cdot 12$ & & $0 \cdot 12$ & $0 \cdot 12$ & $0 \cdot 12$ & $0 \cdot 13$ & & $0 \cdot 12$ & $0 \cdot 00$ \\
\hline Tibia & $0 \cdot 09$ & $0 \cdot 09$ & $0 \cdot 09$ & $0 \cdot 09$ & & $0 \cdot 09$ & $0 \cdot 09$ & $0 \cdot 09$ & $0 \cdot 09$ & & $0 \cdot 09$ & $0 \cdot 09$ & $0 \cdot 08$ & $0 \cdot 09$ & & $0 \cdot 08$ & $0 \cdot 00$ \\
\hline \multicolumn{18}{|l|}{ Other measurements } \\
\hline FMCT $(\mathrm{mm})$ & $2 \cdot 94$ & $3 \cdot 48$ & $3 \cdot 27$ & $3 \cdot 13$ & $*$ & $3 \cdot 02$ & $3 \cdot 23$ & $3 \cdot 20$ & $3 \cdot 58$ & & 3.62 & $3 \cdot 17$ & $3 \cdot 33$ & $3 \cdot 64$ & & $3 \cdot 17$ & $0 \cdot 24$ \\
\hline 12th rib ash $(\mathrm{g} / \mathrm{kg})$ & 528 & 559 & 556 & 552 & $*$ & 535 & 548 & 561 & 556 & & 552 & 556 & 554 & 565 & & 534 & $1 \cdot 04$ \\
\hline 12th $\operatorname{rib}(\mathrm{A}: \mathrm{R})$ & $1 \cdot 12$ & $1 \cdot 27$ & $1 \cdot 25$ & $1 \cdot 24$ & $*$ & $1 \cdot 16$ & $1 \cdot 22$ & 1.28 & $1 \cdot 25$ & & $1 \cdot 23$ & $1 \cdot 27$ & $1 \cdot 24$ & $1 \cdot 31$ & & $1 \cdot 16$ & 0.05 \\
\hline Humerus wt. (g) & $89 \cdot 3$ & $94 \cdot 2$ & $96 \cdot 1$ & $94 \cdot 6$ & & $95 \cdot 3$ & $94 \cdot 4$ & $92 \cdot 6$ & $92 \cdot 2$ & & $85 \cdot 8$ & $82 \cdot 2$ & $90 \cdot 8$ & 91.9 & & $83 \cdot 8$ & $3 \cdot 2$ \\
\hline
\end{tabular}


Table 6. Significance of oestradiol dose responses and effect of sex and castration analysed by orthogonal polynomial contrasts in skeletal measurements of wether $(W)$, ram $(R)$, entire $(E)$ and spayed ewe $(S E)$ lambs

\begin{tabular}{|c|c|c|c|c|c|c|c|c|c|c|}
\hline \multirow[b]{2}{*}{ Parameter } & \multicolumn{2}{|c|}{ Gonadectomy } & \multirow[b]{2}{*}{$\mathrm{W} v . \mathrm{SE}$} & \multicolumn{3}{|c|}{ Linear } & \multicolumn{3}{|c|}{ Quadratic } & \multirow[b]{2}{*}{$\mathrm{R} v \cdot \mathrm{E}$} \\
\hline & $\begin{array}{l}\text { Males } \\
\mathrm{R} v . \mathrm{W}\end{array}$ & $\begin{array}{l}\text { Females } \\
\mathrm{E} v . \mathrm{SE}\end{array}$ & & $\mathrm{W}$ & $\mathrm{R}$ & $\mathrm{SE}$ & $\mathrm{W}$ & $\mathrm{R}$ & $\mathrm{SE}$ & \\
\hline \multicolumn{11}{|l|}{ Bone length } \\
\hline Cannon bone & & & & & $*$ & * & & & $*$ & \\
\hline Gain in cannons & $*$ & & & & $*$ & $*$ & & & $*$ & \\
\hline Total vertebral col. & & $*$ & $*$ & & & $* *$ & & & $*$ & \\
\hline 1st rib & & & $* * *$ & & & & & & & \\
\hline 1st vertebral spine & & & $* * *$ & $*$ & $* * *$ & $*$ & * & $* * *$ & $* *$ & \\
\hline 12 th rib & & & & $* *$ & & $* * *$ & * & & $* *$ & \\
\hline 12 th vertebral spine & $* * *$ & $*$ & & & $*$ & & & $*$ & $*$ & $* * *$ \\
\hline Radius & & & $*$ & & & & & & & \\
\hline Ulna & & & & & & & & & & \\
\hline Humerus & & & $*$ & & & & * & & & \\
\hline Femur & & & & & & & & & & \\
\hline Tibia & $*$ & & & & $*$ & & & & & \\
\hline \multicolumn{11}{|l|}{ Bone diameter } \\
\hline Radius & & & & & $*$ & & & & & \\
\hline Humerus & & & $*$ & $*$ & $*$ & & * & & & \\
\hline Femur & & & & & & $*$ & & & & \\
\hline Tibia & & & & & & & & & & $*$ \\
\hline \multicolumn{11}{|l|}{ Diameter:length ratio } \\
\hline Radius & & $*$ & & & & $* *$ & & & $*$ & \\
\hline Humerus & & & & & & $* *$ & & & & \\
\hline Femur & & & & & & $* *$ & & & & \\
\hline Tibia & & & & & & & & & & \\
\hline \multicolumn{11}{|l|}{ Other measurements } \\
\hline FMCT & & & & & & $*$ & & & $*$ & \\
\hline 12 th rib ash & & & & $*$ & & $* *$ & $*$ & & $*$ & \\
\hline 12th rib $\mathrm{A}: \mathrm{R}$ & & & & $*$ & & $* *$ & $* *$ & & $*$ & \\
\hline Humerus weight & & & $* * *$ & & & & $* * *$ & $* *$ & & $* * *$ \\
\hline
\end{tabular}

$* P<0.05, * * P<0.01, * * * P<0.001$.

Table 7. Coefficients $A, b$ and $k$ (and their standard errors, $S_{A}, S_{b}$ and $S_{k}$ ) from Gompertz equations fitted to mean cannon bone length (adjusted to mean initial cannon bone length by analysis of covariance) and residual standard deviation ( $r s d)$ of control and treated wether, ram, spayed ewe and entire ewe lambs implanted with silicone rubber implants containing low, medium or high doses of oestradiol-17 $\beta(\mathrm{n}=8)(r$ sd $=$ residual standard deviation $)$

\begin{tabular}{|c|c|c|c|c|c|c|c|}
\hline Group & A (antilog) & $\left(\mathrm{S}_{\mathrm{A}}\right)$ & $\mathrm{B}$ & $\left(\mathrm{S}_{\mathrm{b}}\right)$ & $\mathrm{k}$ & $\left(\mathrm{S}_{\mathrm{k}}\right)$ & rsd \\
\hline Control wethers & $161 \cdot 6$ & $6 \cdot 98$ & $0 \cdot 1876$ & $0 \cdot 0417$ & 0.0039 & $0 \cdot 0013$ & $0 \cdot 4967$ \\
\hline Low dose wethers & $156 \cdot 2$ & $7 \cdot 94$ & $0 \cdot 1537$ & $0 \cdot 0488$ & $0 \cdot 0046$ & $0 \cdot 0023$ & $1 \cdot 1061$ \\
\hline Medium dose wethers & $149 \cdot 1$ & $2 \cdot 09$ & $0 \cdot 1041$ & 0.0124 & 0.0092 & 0.0026 & $0 \cdot 7013$ \\
\hline High dose wethers & $147 \cdot 0$ & $2 \cdot 43$ & $0 \cdot 0920$ & $0 \cdot 0149$ & $0 \cdot 0096$ & $0 \cdot 0036$ & $0 \cdot 9419$ \\
\hline Control rams & $149 \cdot 9$ & $3 \cdot 19$ & $0 \cdot 1162$ & 0.0194 & 0.0078 & 0.0027 & $0 \cdot 8855$ \\
\hline Low dose rams & $148 \cdot 7$ & $3 \cdot 80$ & $0 \cdot 1072$ & 0.0235 & 0.0070 & 0.0030 & $0 \cdot 8769$ \\
\hline Medium dose rams & $149 \cdot 2$ & $2 \cdot 53$ & $0 \cdot 1100$ & 0.0156 & 0.0069 & 0.0019 & $0 \cdot 5334$ \\
\hline High dose rams & $150 \cdot 6$ & $3 \cdot 70$ & $0 \cdot 1195$ & 0.0226 & 0.0068 & 0.0024 & $0 \cdot 7828$ \\
\hline Control spayed ewes & $165 \cdot 7$ & $8 \cdot 23$ & $0 \cdot 2114$ & $0 \cdot 0484$ & 0.0034 & $0 \cdot 0011$ & 0.6387 \\
\hline Low dose spayed ewes & $148 \cdot 4$ & $3 \cdot 91$ & $0 \cdot 1069$ & 0.0243 & 0.0072 & 0.0032 & 1.9813 \\
\hline Medium dose spayed ewes & $146 \cdot 6$ & $3 \cdot 15$ & 0.0931 & 0.0196 & 0.0094 & 0.0045 & $0 \cdot 2518$ \\
\hline High dose spayed ewes & $147 \cdot 9$ & $2 \cdot 15$ & $0 \cdot 1013$ & $0 \cdot 0131$ & $0 \cdot 0089$ & 0.0026 & 0.7289 \\
\hline Control entire ewes & $151 \cdot 5$ & $2 \cdot 94$ & $0 \cdot 1243$ & $0 \cdot 0177$ & 0.0075 & 0.0021 & $0 \cdot 7210$ \\
\hline
\end{tabular}


Table 8. Mean length, girth, weight, and girth:length ratio of some dissected muscles of control $(C)$ and treated $(T)$ wether, ram, spayed ewe and entire ewe lambs implanted with low $(L)$, medium $(M)$ or high $(H)$ dose implants containing oestradiol-17 $\beta$

\begin{tabular}{|c|c|c|c|c|c|c|c|c|c|c|c|c|c|c|c|c|c|}
\hline & \multicolumn{4}{|c|}{ Wethers } & \multirow[b]{2}{*}{$\mathrm{C} v . \mathrm{T}$} & \multicolumn{4}{|c|}{ Rams } & \multirow[b]{2}{*}{$\mathrm{C} v . \mathrm{T}$} & \multicolumn{4}{|c|}{ Spayed ewes } & \multicolumn{3}{|c|}{ Entire ewes } \\
\hline & $\mathrm{C}$ & $\mathrm{L}$ & M & $\mathrm{H}$ & & $\mathrm{C}$ & $\mathrm{L}$ & M & $\mathrm{H}$ & & $\mathrm{C}$ & $\mathrm{L}$ & M & $\mathrm{H}$ & $\mathrm{C} v . \mathrm{T}$ & C & ESE \\
\hline \multicolumn{18}{|c|}{ M. biceps brachii (BIC) } \\
\hline Length $(\mathrm{mm})$ & 157 & 159 & 157 & 159 & & 159 & 161 & 164 & 158 & & 156 & 154 & 160 & 157 & & 154 & $2 \cdot 94$ \\
\hline Girth $(\mathrm{mm})$ & $65 \cdot 6$ & $67 \cdot 6$ & $69 \cdot 2$ & $70 \cdot 9$ & $*$ & $70 \cdot 9$ & $70 \cdot 2$ & $70 \cdot 0$ & $71 \cdot 0$ & & $65 \cdot 9$ & $66 \cdot 9$ & $67 \cdot 7$ & $70 \cdot 7$ & $*$ & $66 \cdot 7$ & $1 \cdot 83$ \\
\hline Girth: length ratio & $0 \cdot 42$ & $0 \cdot 43$ & $0 \cdot 44$ & $0 \cdot 45$ & & $0 \cdot 44$ & $0 \cdot 43$ & $0 \cdot 43$ & $0 \cdot 45$ & & $0 \cdot 42$ & $0 \cdot 43$ & 0.43 & $0 \cdot 45$ & & $0 \cdot 43$ & $0 \cdot 01$ \\
\hline Weight $(\mathrm{g})$ & $28 \cdot 7$ & $30 \cdot 3$ & $31 \cdot 7$ & $33 \cdot 1$ & $*$ & $31 \cdot 6$ & $32 \cdot 7$ & $32 \cdot 0$ & $31 \cdot 5$ & & $28 \cdot 2$ & $29 \cdot 3$ & $30 \cdot 5$ & $32 \cdot 9$ & $*$ & $28 \cdot 4$ & $1 \cdot 49$ \\
\hline $\begin{array}{l}\text { Concentration in } \\
\text { carcass }(\mathrm{g} / \mathrm{kg})\end{array}$ & $35 \cdot 6$ & $35 \cdot 3$ & $38 \cdot 5$ & $38 \cdot 4$ & & $38 \cdot 6$ & $40 \cdot 7$ & $38 \cdot 1$ & $36 \cdot 9$ & & $33 \cdot 9$ & $34 \cdot 9$ & $37 \cdot 2$ & $37 \cdot 1$ & $*$ & $35 \cdot 2$ & $0 \cdot 16$ \\
\hline \multicolumn{18}{|c|}{ M. extensor carpi radialis (ECR) } \\
\hline Length (mm) & 180 & 179 & 181 & 177 & & 180 & 177 & 181 & 177 & & 179 & 173 & 173 & 175 & & 176 & $2 \cdot 60$ \\
\hline Girth $(\mathrm{mm})$ & $75 \cdot 2$ & $80 \cdot 4$ & $78 \cdot 9$ & $78 \cdot 0$ & & $80 \cdot 2$ & $78 \cdot 7$ & $76 \cdot 1$ & $77 \cdot 8$ & & $76 \cdot 0$ & $76 \cdot 3$ & $75 \cdot 8$ & $78 \cdot 0$ & & $73 \cdot 5$ & $2 \cdot 49$ \\
\hline Girth: length ratio & 0.42 & $0 \cdot 45$ & $0 \cdot 43$ & $0 \cdot 44$ & & $0 \cdot 45$ & $0 \cdot 44$ & $0 \cdot 42$ & $0 \cdot 44$ & & $0 \cdot 42$ & $0 \cdot 44$ & $0 \cdot 44$ & $0 \cdot 44$ & & $0 \cdot 42$ & $0 \cdot 01$ \\
\hline Weight $(\mathrm{g})$ & $32 \cdot 6$ & $36 \cdot 4$ & $36 \cdot 0$ & $35 \cdot 8$ & & $38 \cdot 0$ & $37 \cdot 6$ & $36 \cdot 1$ & $35 \cdot 7$ & & $34 \cdot 6$ & $32 \cdot 0$ & $32 \cdot 4$ & $36 \cdot 4$ & & $34 \cdot 3$ & $2 \cdot 14$ \\
\hline $\begin{array}{l}\text { Concentration in } \\
\text { carcass }(\mathrm{g} / \mathrm{kg})\end{array}$ & $44 \cdot 3$ & $42 \cdot 4$ & $43 \cdot 7$ & $41 \cdot 4$ & & $46 \cdot 2$ & $46 \cdot 4$ & $42 \cdot 8$ & $41 \cdot 8$ & & $41 \cdot 6$ & $38 \cdot 1$ & $39 \cdot 3$ & $41 \cdot 1$ & & $42 \cdot 4$ & $0 \cdot 02$ \\
\hline \multicolumn{18}{|l|}{ M. splenius } \\
\hline Weight (g) & $7 \cdot 5$ & $7 \cdot 3$ & $7 \cdot 4$ & $9 \cdot 6$ & & $15 \cdot 4$ & $13 \cdot 9$ & $10 \cdot 4$ & $9 \cdot 8$ & & $5 \cdot 6$ & $7 \cdot 5$ & $6 \cdot 5$ & $7 \cdot 1$ & & $6 \cdot 0$ & $1 \cdot 70$ \\
\hline $\begin{array}{l}\text { Concentration in } \\
\text { carcass }(\mathrm{g} / \mathrm{kg})\end{array}$ & $9 \cdot 6$ & $8 \cdot 7$ & $9 \cdot 3$ & $11 \cdot 2$ & & $18 \cdot 5$ & $17 \cdot 0$ & $12 \cdot 0$ & $11 \cdot 3$ & $*$ & $6 \cdot 7$ & $8 \cdot 9$ & $8 \cdot 0$ & $8 \cdot 1$ & & $7 \cdot 5$ & $0 \cdot 19$ \\
\hline
\end{tabular}

$* P<0 \cdot 05$. 
Table 9. Significance of effect of gonadectomy, oestradiol dose response and sex on muscle data analysed by orthogonal polynomial contrasts in wether $(W)$, ram $(R)$, entire ewe $(E)$ and spayed ewe $(S E)$ lambs

\begin{tabular}{|c|c|c|c|c|c|c|c|c|c|c|}
\hline & \multicolumn{2}{|c|}{ Gonadectomy } & \multirow[b]{2}{*}{$\mathrm{W} v . \mathrm{SE}$} & \multicolumn{3}{|c|}{ Linear } & \multicolumn{3}{|c|}{ Quadratic } & \multirow[b]{2}{*}{$\mathrm{R} v . \mathrm{E}$} \\
\hline & $\mathrm{R} v . \mathrm{W}$ & E v. SE & & $\mathrm{W}$ & $\mathrm{R}$ & SE & W & $\mathrm{R}$ & SE & \\
\hline \multicolumn{11}{|l|}{ M. biceps brachii (BIC) } \\
\hline Length & & & & & $*$ & & & ** & & \\
\hline Girth & ** & & & & ** & & ** & $*$ & & * \\
\hline Girth: length ratio & & & & & & & * & & & \\
\hline Weight & & & & & * & & $* * *$ & ** & & * \\
\hline \multicolumn{11}{|c|}{ Proportion of carcass } \\
\hline \multicolumn{11}{|c|}{ M. extensor carpi radialis (ECR) } \\
\hline Length & & $* * *$ & & $*$ & & ** & & & $* * *$ & \\
\hline Girth & * & * & & & & & & & & ** \\
\hline Girth:length ratio & & & & & & & & & & \\
\hline Weight & * & * & & & & & & * & & \\
\hline \multicolumn{11}{|l|}{ Proportion of carcass } \\
\hline M. splenius & & & & & & & & & & \\
\hline Weight & $* * *$ & ** & & $* * *$ & *** & & & & ** & $* * *$ \\
\hline Proportion of carcass & & & & & & & & & & \\
\hline
\end{tabular}

$* P<0.05, * * P<0.01, * * * P<0.001$.

pooled and analysed using orthogonal polynomial contrasts. The results, which are shown in Tables 12 and 13 , substantiate the inhibitory effect of oestradiol treatment on growth of cannon bones and its stimulatory effects on the vertebral column, 12th rib, BIC muscle and ECR muscle in these animals.

Oestradiol treatment increased teat length, carcass protein and water content and decreased fat content in the wether and spayed ewe lambs (Tables 10 and 11). In addition, spayed ewe lambs treated with oestradiol had significantly longer teats, heavier warm carcass, head, and uteri weights, and had less fat and more protein and water content in the carcass than their controls (Tables 10 and 11).

\section{Effects of oestradiol implants - entire ram lambs}

Treatment of ram lambs with oestradiol had some minor stimulatory effects on skeletal development (Table 5 and 6), it reduced weight of the splenius muscle (Tables 8 and 9) and testes, and it increased teat length (Tables 10 and 11).

\section{DISCUSSION}

These results show that the effects of gender and sex steroids on musculo-skeletal growth of sheep vary for different regions of the body, probably depending on the relative state of maturity of each region. In general, entire males had heavier muscles and distal limb bones than females, and oestradiol treatment of rams, wethers and spayed ewes stimulated growth of central skeletal regions but inhibited growth in distal limbs. This can be interpreted as an acceleration of the differential maturation of the skeleton where the central skeletal core ceases to increase in size later than the distal limbs. In the current study this effect of maleness and oestradiol was manifested as a comparative elongation of the vertebral column and an inhibition of growth (early cessation) of the cannon bones and associated muscles.

\section{Effectiveness of oestradiol implants}

Loss of weight by the oestradiol-containing implants and plasma oestradiol concentrations indicated that treated animals had received substantial amounts of oestradiol during the experimental period. This was borne out by increases in teat length and uterine weight and the reduction of testes weight in the implanted lambs.

\section{Effects of exogenous oestrogens}

In the present study the high dose of oestradiol resulted in an increase of $19 \%, 8 \%$ and $21 \%$ live weight in treated wether, ram and spayed ewes, respectively, above that of their controls. These values are comparable to those of Muir (1985) who stated that oestrogens may stimulate live-weight gain by $10-20 \%$ in ruminants. However, gains in live weight in the present experiment were not exceptionally high $(159 \mathrm{~g} /$ day maximum) compared with other data for growth rates of lambs on pasture (e.g. $230 \mathrm{~g} /$ day, Everest \& Scales 1983). In experiments which have reported greater stimulation in live weight of lambs treated with oestradiol than recorded here, high quality feeds were used. For example Galbraith et al. 
Table 10. Mean fresh weights of some non-carcass components, carcass measurements and carcass chemical composition values $(\mathrm{n}=8)$ and difference between control $(C)$ and treated $(T)$ wether, ram, spayed ewe and entire ewe lambs implanted with low $(L)$, medium $(M)$ or high $(H)$ dose silicone implants containing oestradiol-17 $\beta$

\begin{tabular}{|c|c|c|c|c|c|c|c|c|c|c|c|c|c|c|c|c|c|}
\hline & \multicolumn{4}{|c|}{ Wethers } & \multirow[b]{2}{*}{$\mathrm{C} v . \mathrm{T}$} & \multicolumn{4}{|c|}{ Rams } & \multirow[b]{2}{*}{$\mathrm{C} v . \mathrm{T}$} & \multicolumn{4}{|c|}{ Spayed ewes } & \multirow{2}{*}{\multicolumn{2}{|c|}{$\mathrm{C} v . \mathrm{T} \quad \mathrm{C}^{\text {Entire ewes }}$}} & \multirow[b]{2}{*}{ ESE } \\
\hline & $\mathrm{C}$ & $\mathrm{L}$ & M & $\mathrm{H}$ & & $\mathrm{C}$ & $\mathrm{L}$ & M & $\mathrm{H}$ & & $\mathrm{C}$ & $\mathrm{L}$ & M & $\mathrm{H}$ & & & \\
\hline Teat length (mm) & $8 \cdot 5$ & $17 \cdot 7$ & $17 \cdot 4$ & $17 \cdot 0$ & $* * *$ & $8 \cdot 2$ & $13 \cdot 7$ & $15 \cdot 2$ & $16 \cdot 9$ & $* * *$ & $9 \cdot 9$ & $12 \cdot 9$ & $16 \cdot 7$ & $17 \cdot 9$ & $* * *$ & $12 \cdot 5$ & $1 \cdot 1$ \\
\hline Final live weight (kg) & $39 \cdot 4$ & $42 \cdot 6$ & $40 \cdot 7$ & $43 \cdot 0$ & & $41 \cdot 7$ & $40 \cdot 4$ & $42 \cdot 6$ & $43 \cdot 2$ & & $40 \cdot 4$ & $40 \cdot 5$ & $42 \cdot 8$ & $44 \cdot 7$ & $* *$ & $40 \cdot 0$ & $1 \cdot 3$ \\
\hline Live-weight gain (g/day) & 124 & 145 & 133 & 147 & & 139 & 130 & 145 & 150 & & 131 & 132 & 147 & 159 & $* *$ & 128 & $7 \cdot 6$ \\
\hline Warm carcass weight $(\mathrm{kg})$ & $16 \cdot 9$ & $18 \cdot 2$ & $17 \cdot 1$ & $17 \cdot 9$ & & $17 \cdot 4$ & $17 \cdot 1$ & $18 \cdot 0$ & $18 \cdot 2$ & & $17 \cdot 3$ & $17 \cdot 5$ & $17 \cdot 5$ & $18 \cdot 8$ & $* *$ & $17 \cdot 1$ & $0 \cdot 7$ \\
\hline \multicolumn{18}{|l|}{ Organ weight $(\mathrm{g})$} \\
\hline Head & 2250 & 2420 & 2400 & 2310 & & 2460 & 2460 & 2550 & 2540 & $*$ & 2170 & 2070 & 2340 & 2480 & $*$ & 2190 & 90 \\
\hline Testes & & & & & & 339 & 246 & 118 & 97 & $* * *$ & & & & & & $4 \cdot 1$ & $2 \cdot 7$ \\
\hline Uterus & & & & & & & & & & & $10 \cdot 0$ & $19 \cdot 0$ & $22 \cdot 7$ & $19 \cdot 9$ & $* * *$ & $32 \cdot 3$ & 2 \\
\hline Fat depot weight (g) & & & & & & & & & & & & & & & & & 150 \\
\hline Total non-carcass fat & 1389 & 1376 & 1207 & 1175 & & 1267 & 1066 & 1255 & 1142 & & 1739 & 1505 & 1392 & 1473 & * & 1585 & \\
\hline \multicolumn{18}{|l|}{ Carcass measurements $(\mathrm{mm})$} \\
\hline GR measurement & $8 \cdot 6$ & $9 \cdot 3$ & $5 \cdot 7$ & $7 \cdot 5$ & & $6 \cdot 7$ & $5 \cdot 3$ & $8 \cdot 5$ & $8 \cdot 5$ & $*$ & $10 \cdot 2$ & $9 \cdot 6$ & $6 \cdot 5$ & $8 \cdot 4$ & & $8 \cdot 7$ & $1 \cdot 3$ \\
\hline LD A measurement & $51 \cdot 1$ & $54 \cdot 4$ & $53 \cdot 2$ & $52 \cdot 6$ & & $54 \cdot 6$ & $54 \cdot 0$ & $53 \cdot 3$ & $52 \cdot 8$ & & $53 \cdot 0$ & $51 \cdot 4$ & $54 \cdot 8$ & $54 \cdot 8$ & & $53 \cdot 7$ & $1 \cdot 5$ \\
\hline LD B measurement & $23 \cdot 3$ & $24 \cdot 9$ & $24 \cdot 3$ & $23 \cdot 8$ & & $24 \cdot 6$ & $22 \cdot 8$ & $23 \cdot 9$ & $23 \cdot 9$ & & $25 \cdot 2$ & $23 \cdot 7$ & $24 \cdot 4$ & $27 \cdot 2$ & & $24 \cdot 7$ & $1 \cdot 4$ \\
\hline $\mathrm{C}$ measurement & $5 \cdot 4$ & $4 \cdot 1$ & $2 \cdot 6$ & $3 \cdot 7$ & $*$ & $3 \cdot 4$ & $3 \cdot 0$ & $4 \cdot 1$ & $4 \cdot 5$ & & $5 \cdot 1$ & $5 \cdot 0$ & $3 \cdot 7$ & $3 \cdot 8$ & & $4 \cdot 8$ & $0 \cdot 9$ \\
\hline \multicolumn{18}{|l|}{$\begin{array}{l}\text { Chemical composition } \\
\text { (g/kg on DM basis) }\end{array}$} \\
\hline Carcass fat & 570 & 552 & 499 & 516 & $* *$ & 524 & 492 & 533 & 533 & & 573 & 583 & 521 & 516 & $* *$ & 576 & $1 \cdot 75$ \\
\hline Carcass protein & 340 & 351 & 395 & 379 & $* *$ & 381 & 408 & 366 & 370 & $*$ & 333 & 334 & 378 & 384 & $* *$ & 336 & $1 \cdot 4$ \\
\hline Carcase water & 513 & 525 & 541 & 543 & $*$ & 540 & 551 & 519 & 534 & & 500 & 508 & 549 & 540 & $* *$ & 510 & $1 \cdot 2$ \\
\hline
\end{tabular}

$* P<0 \cdot 05, * * P<0 \cdot 01, * * * P<0 \cdot 001$. 
Table 11. Significance of oestradiol dose responses and effect of sex and castration analysed by orthogonal polynomial contrasts in live weight growth and body composition of wether $(W)$, ram $(R)$, entire $(E)$ and spayed ewe (SE) lambs

\begin{tabular}{|c|c|c|c|c|c|c|c|c|c|c|}
\hline \multirow[b]{2}{*}{ Parameter } & \multicolumn{2}{|c|}{ Gonadectomy } & \multirow[b]{2}{*}{$\mathrm{W} v . \mathrm{SE}$} & \multicolumn{3}{|c|}{ Linear } & \multicolumn{3}{|c|}{ Quadratic } & \multirow[b]{2}{*}{$\mathrm{R} v . \mathrm{E}$} \\
\hline & $\begin{array}{l}\text { Males } \\
\mathrm{R} v . \mathrm{W}\end{array}$ & $\begin{array}{l}\text { Females } \\
\text { E } v \text {. SE }\end{array}$ & & $\mathrm{W}$ & $\mathrm{R}$ & SE & $\mathrm{W}$ & $\mathrm{R}$ & SE & \\
\hline Final teat length & & $*$ & & $* * *$ & & $* * *$ & $* * *$ & ** & $* * *$ & $* * *$ \\
\hline Final live weight & & & & & & $* * *$ & $* *$ & & $* * *$ & \\
\hline Warm carcass weight & & & & & & & & & * & \\
\hline Head weight & * & & * & & $* * *$ & & & $* * *$ & & ** \\
\hline Total non-carcass fat weight & & & ** & * & $* * *$ & * & & ** & & * \\
\hline Testes weight & & & & & & & & ** & & \\
\hline Uterus weight & & $* * *$ & & & & & & & & \\
\hline GR measurement & & & & & & & & ** & & \\
\hline LD A measurement & $*$ & & & & & & & & & \\
\hline LD B measurement & & & & & & & & & * & \\
\hline $\mathrm{C}$ measurement & $*$ & & & & & & * & $* *$ & & \\
\hline Carcass fat & * & & * & & * & & $* * *$ & $* * *$ & & ** \\
\hline Carcass protein & ** & & & & * & & $* * *$ & $* * *$ & & ** \\
\hline Carcass water & $*$ & & & & & & $* * *$ & ** & & * \\
\hline
\end{tabular}

* $P<0.05, * * P<0.01, * * * P<0 \cdot 001$.

(1990) used a pelleted concentrate feed and reported live-weight gains of $257 \mathrm{~g} /$ day in treated and $206 \mathrm{~g} /$ day in control wether lambs; a $25 \%$ increase in the treated group.

Reports in the literature on the effects of oestradiol on live-weight growth of sheep are inconsistent but are often based on dissimilar experimental conditions. For instance Galbraith et al. (1990) treated lambs at $18 \mathrm{~kg}$ (approximately 10 weeks old), whereas in studies reported by Hunter et al. (1987) and Bass et al. (1989), animals were treated from 4-6 weeks of age. Therefore, contradictory responses to oestradiol treatment may arise from differences in ages (stages of maturity) of animals. Growth rate in sheep increases sharply in early postnatal life, reaching a maximum when the animal achieves about $20 \%$ of its mature weight and declines thereafter (Butterfield 1988). Other studies have shown that oestrogens are not very effective in stimulating live-weight gain in lambs of $0-5$ months old (J. J. Bass, personal communication). It is possible to speculate that treatment with oestrogens in early postnatal life has little effect on live-weight growth simply because these animals are already growing at a high rate. However, with the progress of maturity, i.e. when animals have heavier weights and growth rates slow down, effects of treatment with oestrogens or other growth promoters may become more pronounced.

Oestradiol treatment reduced weight of carcass fat and non-carcass fat, especially in wether and spayed female lambs, which is in agreement with other studies on fat deposition in ruminants (Galbraith \& Topps 1981; Bass et al. 1989). The latter authors proposed the use of oestrogens in sheep not only to promote growth but also to prevent overfatness in lambs. The present study confirms this view and indicates that increased live-weight gain also could be achieved through the use of oestrogens in pasture-fed sheep. Lack of a fat-reducing effect of oestradiol in the rams can be explained by the fact that they were leaner than the other lambs to start with; the fat levels in spayed ewes and wethers only approaching values as low as those of the rams at the high dose oestradiol treatment.

It is possible that compounds with sex steroidal activity were present in the pasture consumed by lambs in this study, either as naturally occurring phyto-oestrogens or from excretion of oestradiol or its metabolites by the lambs which were treated with the oestradiol-containing implants. However, this concern can be largely allayed by the data for teat length in non-implanted wether lambs (mean = $8.5 \mathrm{~mm}$, Table 10) which are similar to those (mean $=$ $8.2 \mathrm{~mm}$ ) of wether lambs at equivalent live weights which had not been exposed to oestrogens in the study reported by Galbraith et al. (1997). The sensitivity of this parameter to oestrogens is well demonstrated by the lengths recorded in the oestradiol-treated wethers in the present study (see Table 10).

\section{Effects of sex and gonadectomy}

Increased growth in length of cannon bones following castration suggests that lack of androgens may have delayed the epiphyseal closure of these early maturing 
Table 12. Skeletal data (means, $\mathrm{n}=8$ ) of non-implanted lambs $(C)$ and lambs $(T)$ implanted with silicone rubber implants containing different doses $(L, M, H)$ of oestradiol (data pooled from wether and spayed ewe lambs); comparison between control and treated lambs, and response to dose of oestradiol in treated lambs

\begin{tabular}{|c|c|c|c|c|c|c|c|}
\hline & \multicolumn{4}{|c|}{ Dose } & \multirow[b]{2}{*}{ ESE } & \multirow[b]{2}{*}{$\mathrm{C} v . \mathrm{T}$} & \multirow[b]{2}{*}{ Dose response $\dagger$} \\
\hline & $\mathrm{C}$ & $\mathrm{L}$ & M & $\mathrm{H}$ & & & \\
\hline \multicolumn{8}{|l|}{ Bone length (mm) } \\
\hline Cannon bones final & 147 & 144 & 144 & 144 & $0 \cdot 8$ & $* * *$ & \\
\hline Gain in cannon bones & 13 & 11 & 10 & 10 & $0 \cdot 8$ & $* * *$ & \\
\hline Total vertebral column & 812 & 820 & 826 & 838 & $9 \cdot 1$ & $*$ & \\
\hline lst rib & 94 & 94 & 96 & 95 & $2 \cdot 1$ & & \\
\hline 1st vertebral spine & 42 & 43 & 42 & 42 & $1 \cdot 4$ & & \\
\hline 12 th rib & 179 & 183 & 187 & 191 & $3 \cdot 4$ & $*$ & $*$ \\
\hline 12 th vertebral spine & 22 & 22 & 22 & 23 & $0 \cdot 7$ & & \\
\hline Radius & 137 & 136 & 137 & 136 & $0 \cdot 9$ & & \\
\hline Ulna & 169 & 170 & 170 & 167 & $1 \cdot 4$ & & \\
\hline Humerus & 120 & 120 & 122 & 121 & $1 \cdot 0$ & & \\
\hline Femur & 154 & 154 & 155 & 156 & $1 \cdot 4$ & & \\
\hline Tibia & 177 & 179 & 178 & 179 & $1 \cdot 4$ & & \\
\hline \multicolumn{8}{|l|}{ Bone diameter (mm) } \\
\hline Radius & $17 \cdot 0$ & $17 \cdot 4$ & $17 \cdot 5$ & $17 \cdot 9$ & $0 \cdot 4$ & $*$ & \\
\hline Humerus & $18 \cdot 3$ & $19 \cdot 0$ & $19 \cdot 3$ & $19 \cdot 6$ & $0 \cdot 4$ & $* *$ & \\
\hline Femur & $18 \cdot 4$ & $19 \cdot 0$ & $19 \cdot 3$ & $19 \cdot 4$ & $0 \cdot 4$ & $*$ & \\
\hline Tibia & $15 \cdot 2$ & $15 \cdot 2$ & $15 \cdot 3$ & $15 \cdot 7$ & $0 \cdot 2$ & & \\
\hline \multicolumn{8}{|l|}{ Diameter:length ratio } \\
\hline Radius & $0 \cdot 124$ & $0 \cdot 127$ & $0 \cdot 128$ & $0 \cdot 132$ & $0 \cdot 003$ & $*$ & \\
\hline Humerus & $0 \cdot 153$ & $0 \cdot 157$ & $0 \cdot 158$ & $0 \cdot 162$ & $0 \cdot 003$ & $*$ & \\
\hline Femur & $0 \cdot 120$ & $0 \cdot 123$ & $0 \cdot 125$ & $0 \cdot 125$ & 0.003 & $*$ & \\
\hline Tibia & $0 \cdot 086$ & $0 \cdot 085$ & 0.086 & 0.087 & $0 \cdot 001$ & & \\
\hline \multicolumn{8}{|l|}{ Other measurements } \\
\hline Humerus weight (g) & $87 \cdot 4$ & $88 \cdot 0$ & $93 \cdot 4$ & $93 \cdot 0$ & $2 \cdot 8$ & & \\
\hline $\mathrm{FMCT}(\mathrm{mm})$ & $3 \cdot 3$ & $3 \cdot 3$ & $3 \cdot 3$ & $3 \cdot 4$ & $0 \cdot 16$ & & \\
\hline 12 th rib ash $(\mathrm{g} / \mathrm{kg})$ & 540 & 557 & 555 & 559 & 0.66 & $* *$ & \\
\hline 12 th $\operatorname{rib}(A: R)$ & $1 \cdot 178$ & $1 \cdot 262$ & $1 \cdot 249$ & $1 \cdot 274$ & $0 \cdot 032$ & $* *$ & \\
\hline \multicolumn{8}{|l|}{ Muscle measurements } \\
\hline \multicolumn{8}{|l|}{ M. biceps brachii (BIC) } \\
\hline Length $(\mathrm{mm})$ & 156 & 156 & 158 & 158 & $2 \cdot 24$ & & \\
\hline Girth $(\mathrm{mm})$ & 66 & 67 & 68 & 71 & $1 \cdot 16$ & $* *$ & $* *$ \\
\hline Girth: length ratio & $0 \cdot 422$ & $0 \cdot 431$ & $0 \cdot 435$ & $0 \cdot 449$ & $0 \cdot 01$ & $*$ & \\
\hline Weight $(\mathrm{g})$ & $28 \cdot 4$ & $29 \cdot 8$ & $31 \cdot 1$ & $32 \cdot 9$ & $1 \cdot 00$ & $* * *$ & $* *$ \\
\hline Concentration in carcass $(\mathrm{g} / \mathrm{kg})$ & $3 \cdot 5$ & $3 \cdot 5$ & $3 \cdot 8$ & $3 \cdot 8$ & $0 \cdot 01$ & $*$ & $*$ \\
\hline \multicolumn{8}{|l|}{ M. extensor carpi radialis (ECR) } \\
\hline Length $(\mathrm{mm})$ & 179 & 176 & 177 & 176 & $2 \cdot 07$ & & \\
\hline Girth $(\mathrm{mm})$ & 76 & 78 & 77 & 78 & 174 & & \\
\hline Girth:length ratio & $0 \cdot 421$ & $0 \cdot 444$ & $0 \cdot 436$ & $0 \cdot 443$ & $0 \cdot 01$ & $*$ & \\
\hline Weight $(\mathrm{g})$ & $33 \cdot 5$ & $34 \cdot 1$ & $34 \cdot 3$ & $36 \cdot 0$ & $1 \cdot 50$ & & \\
\hline Concentration in carcass $(\mathrm{g} / \mathrm{kg})$ & $4 \cdot 1$ & $4 \cdot 0$ & $4 \cdot 3$ & $4 \cdot 1$ & $0 \cdot 01$ & & \\
\hline \multicolumn{8}{|l|}{ M. splenius } \\
\hline Weight $(\mathrm{g})$ & $6 \cdot 5$ & $7 \cdot 4$ & $7 \cdot 0$ & $8 \cdot 3$ & $0 \cdot 80$ & & \\
\hline Concentration in carcass $(\mathrm{g} / \mathrm{kg})$ & $0 \cdot 82$ & $0 \cdot 87$ & $0 \cdot 87$ & 0.95 & $0 \cdot 01$ & & \\
\hline
\end{tabular}

FMCT, femur maximum cortical thickness; ESE, estimated standard error.

* $P<0.05, * * P<0.01$, *** $P<0.001$.

$\dagger$ Only linear dose responses shown, quadratic responses are all non-significant.

limb bones. Bones of castrated male lambs were also smaller in diameter which resulted in lower values for their diameter:length ratios. The decrease in cortical thickness, bone weight and ash proportion indicates retardation of bone deposition and mineralization.
Stimulation of skeletal growth of ewe lambs as a result of spaying was not marked but was concordant with previous findings on growth of distal limbs in spayed heifers (Hubard Ocariz et al. 1970; Brannang $1971 b$ ). 
Table 13. Means of growth and body composition parameters $(\mathrm{n}=8)$ of non-implanted lambs $(C)$ and lambs $(T)$ implanted with silicone rubber implants containing different doses $(L, M, H)$ of oestradiol-17 $\beta$ (data pooled from wether and spayed ewe lambs); comparison between control and treated lambs, and response to dose of oestradiol in treated lambs

\begin{tabular}{|c|c|c|c|c|c|c|c|c|}
\hline & \multicolumn{4}{|c|}{ Dose } & \multirow[b]{2}{*}{ ESE } & \multirow[b]{2}{*}{ C v. T } & \multicolumn{2}{|c|}{ Dose response } \\
\hline & $\mathrm{C}$ & $\mathrm{L}$ & M & $\mathrm{H}$ & & & Linear & Quadratic \\
\hline Final teat length $(\mathrm{mm})$ & $9 \cdot 18$ & $15 \cdot 20$ & $16 \cdot 94$ & $17 \cdot 31$ & $0 \cdot 70$ & $* * *$ & $* * *$ & \\
\hline Final live weight (kg) & $39 \cdot 84$ & $41 \cdot 47$ & $41 \cdot 60$ & $43 \cdot 79$ & $0 \cdot 97$ & $* *$ & * & \\
\hline Live-weight gain (g/day) & 127 & 138 & 139 & 154 & 6 & ** & $*$ & \\
\hline Warm carcass weight $(\mathrm{kg})$ & $17 \cdot 02$ & $17 \cdot 80$ & $17 \cdot 27$ & $18 \cdot 34$ & 0.05 & & & \\
\hline Head weight $(\mathrm{g})$ & 2200 & 2240 & 2370 & 2400 & 70 & * & * & \\
\hline Total non-carcass fat weight & 1566 & 1436 & 1294 & 1332 & 117 & * & & \\
\hline GR measurement & $9 \cdot 4$ & $9 \cdot 4$ & $6 \cdot 1$ & $8 \cdot 7$ & $1 \cdot 0$ & & & \\
\hline LD A measurement & 52 & 53 & 54 & 54 & $1 \cdot 0$ & & & \\
\hline LD B measurement & 24 & 24 & 24 & 26 & $1 \cdot 0$ & & & \\
\hline $\mathrm{C}$ measurement & $5 \cdot 3$ & $4 \cdot 6$ & $3 \cdot 1$ & $3 \cdot 7$ & 0.6 & $* *$ & & $*$ \\
\hline Carcass fat $(\mathrm{g} / \mathrm{kg})$ & 572 & 567 & 509 & 516 & $1 \cdot 2$ & $* * *$ & $* * *$ & ** \\
\hline Carcass protein $(\mathrm{g} / \mathrm{kg})$ & 337 & 343 & 387 & 381 & $1 \cdot 0$ & $* * *$ & $* * *$ & ** \\
\hline Carcass water $(\mathrm{g} / \mathrm{kg})$ & 507 & 516 & 540 & 541 & $0 \cdot 8$ & $* * *$ & $* *$ & \\
\hline
\end{tabular}

ESE, estimated standard error.

$* P<0.05, * * P<0.01, * * * P<0.001$.

Effects of castration in reducing muscular growth in male lambs were especially marked in the splenius muscle which has been observed previously (Brannang $1971 b$ ). In addition splenius muscle development was inhibited by oestradiol treatment in entire ram lambs. This muscle has been recognized as a target tissue for sex hormones in males of some species. For example, it showed a marked increase in size prior to the rut in deer stags (Tan \& Fennessy 1981; Field et al. 1985). Seasonal changes in this muscle appear to be positively related to blood levels of testosterone in male deer (Field et al. 1985) which may explain the negative effect of castration on this muscle. Also the high dose of oestradiol used in this experiment may have inhibited secretion of luteinising hormone $(\mathrm{LH})$ and consequently reduced blood levels of testosterone. This is supported by the marked reduction in weight of testes which could be attributed to lowered secretion of $\mathrm{LH}$ as a consequence of the negative feedback action of oestradiol (Shanbacher \& Ford 1977). Thus testosterone may be regarded as an essential factor in determining the large splenius weight in rams and the response of this muscle to oestradiol could be explained by a reduction in the secretion of testosterone.

Factors that determine sexual dimorphism in mammals are not well understood although they involve both genetic and hormonal mechanisms (Short 1980). In the present experiment, lack of androgens affected growth of sheep more than lack of oestrogens. However, it was not possible to define a critical age at which sex hormones have most effect on growth.

\section{Effects of oestrogens in gonadectomized animals}

In this experiment, oestradiol treatment generally inhibited longitudinal growth of distal limb bones (cannon bones), stimulated that of vertebral column and ribs, but had little effect on linear growth of medial and proximal limb bones. There are few other reports of effects of oestrogens on specific bones in sheep. For example, oestradiol-17 $\beta$ implants increased rib size in lambs (Galbraith et al. 1997). Stimulation on the one hand and inhibition on the other of bone growth following administration of oestrogens has been explained by the premise that low doses of oestrogens stimulate longitudinal bone growth, while large doses inhibit it (Suzuki 1958; Silberberg \& Silberberg 1972; Short 1980). In light of the results of the present study this explanation can no longer be supported. Firstly, the effects of oestradiol which were recorded here were generally linearly doserelated. Secondly, studies in meat animals show a disto-proximal and cranio-caudal pattern of skeletal maturation which results in differential maturity of the different regions of the skeleton (reviewed by Davies et al. 1984). Consequently, an interpretation of the present results is that oestradiol has accelerated closure of epiphyseal plates of early maturing bones (cannon bones), so that further elongation was limited, and it has stimulated longitudinal growth of late maturing bones (vertebral column and rib) where it did not cause premature closure of the epiphyseal plates. Differential growth patterns in normally growing skeletons were attributed to differences in rates of cell division or to differences in cartilage cell 
population in epiphyseal plates (Burwell 1986). In an actively growing bone, oestrogens, which have anabolic effects, may increase the rate of cell division, thus producing longer bones. In bones approaching maturity such stimulation accelerates cessation of epiphyseal plate activity and will curtail elongation, i.e. cause a relative shortening of bones. Oestrogenic effects on bone may also be modified by the differential regulation of steroid receptors. For instance, in active bones, oestrogen receptors may be upregulated in comparison with those in bones where epiphyseal activity is slowing down.

In the present experiment there was little effect of oestradiol on final length of limb bones, which is in contrast to the effects reported previously (Wilkinson et al. 1955; Shroder \& Hansard 1959). However, data from serial radiographs (not presented) indicated that the tibia bone was longer in oestradiol-treated wethers than controls at days 61 and 134 . This suggests that there may have been early stimulation of linear growth by the hormone, the effect of which had disappeared towards the end of the trial.

In contrast to its minimal effects on their length, oestradiol tended to increase diameter (and thus, diameter: length ratio) and cortical thickness of limb bones, with the effect being significant in the case of the femur. These findings suggest that stimulation of periosteal growth was not accompanied by an equivalent amount of bone resorption on the medullary surface. General inhibition of bone resorption by oestrogens is recognized as a classic effect of these compounds and, thus, they are widely utilized for the treatment of osteoporosis in humans.

Treatment with oestradiol produced little effect on muscle length. This may be explained by the lack of effect on length of limb bones to which these muscles were attached. Growth in length of muscles follows that of intimately related bones (Hooper 1978 $a, b$ ). In contrast to the lack of response of muscle length, girth of $m$. biceps brachii and consequently girth:length ratio were stimulated by oestradiol treatment which must have altered the shape of this muscle, i.e. made it relatively thicker for its length. Such remodelling of muscle shape may result from the direct anabolic effects of oestrogens on skeletal muscle (Galbraith \& Topps 1981).

In the present experiment the most marked stimulatory effects of oestrogens on bone growth (especially on linear growth of vertebrae) were in spayed ewe lambs, more so than in wethers, whereas entire males showed little response at all. Some studies of the effects of oestrogens on live-weight growth in sheep have indicated that castrated male ruminants are the most responsive to oestrogen treatment (Galbraith \& Topps 1981; Muir 1985; Hancock et al. 1991). However, according to Bradfield (1968), animals of different sexes take different growth routes to reach maturity. Consequently, variation in response to oestrogens may result from applying the treatment to animals of the same age but at different stages of maturity. In addition, Bradfield (1968) reported that exogenous oestrogens had more pronounced effects on growth at the lower slaughter weights $(36.3 \mathrm{~kg})$ where lambs of all sex/castration combinations except the entire males responded, whereas the spayed ewes were the only group of lambs to respond to treatment at the higher weight $(45 \cdot 4 \mathrm{~kg})$. The study of Bradfield (1968) is concordant in both respects (sex class of responders and live weight at slaughter) with the present case and has a similar result.

On the basis of the results described in the present paper, it may be concluded that the sex steroids have an essential role in controlling skeletal growth in sheep. However, the effects of these hormones need to be interpreted with attention to degree of maturity of animals, differential pattern of skeletal maturation and dose of the hormone. Growth of early maturing parts of the skeleton was inhibited by the administration of exogenous oestrogens whereas that of late maturing parts of the skeleton was stimulated.

We are grateful to Drs J. R. Sedcole and M. J. Young for their advice on statistical analyses.

\section{REFERENCES}

Alvey, N. G. et al. (1980). GENSTAT: a General Statistical Program. Lawes Agricultural Trust (Rothamsted Experimental Station). Parts I \& II.

AOAC (1984). Official Methods of Analysis. 14th edition. Virginia: Association of Official Analytical Chemists.

Bass, J. J., Fowke, P. J., Duganzich, D. M. \& Peterson, A. J. (1989). Effects of different doses of $17 \beta$-oestradiol on growth and carcass composition of wether and ewe lambs. Journal of Agricultural Science, Cambridge 113, 183-187.

BRADFIELD, P. G. E. (1968). Sex differences in the growth of sheep. In Growth and Development of Mammals (Eds G. A. Lodge \& G. E. Lamming), pp. 92-108. London: Butterworths.

BranNANG, E. (1971a). Studies on monozygous cattle twins XXIII. The effect of castration and age of castration on the development of single muscles, bones and special sex characters. Part II. Swedish Journal of Agricultural Research 1, 69-78.

BRANNANG, E. (1971b). Studies on monozygous cattle twins. XXIV. Some notes on the effect of ovariectomy. Swedish Journal of Agricultural Research 1, 79-82.

Burwell, R. G. (1986). The growth of bone. In Control and Manipulation of Animal Growth (Eds P. J. Buttery, N. B. Haynes \& D. B. Lindsay), pp. 53-65. London: Butterworths.

Butterfield, R. M. (1988). New Concepts of Sheep Growth. University of Sydney, Australia.

DAvies, A. S. (1979). Musculoskeletal growth gradients: A contribution to quadrupedal mechanics. Anatomia Histologia Embryologia 8, 164-167. 
Davies, A. S., TAN, G. Y. \& Broad, T. F. (1984). Growth gradients in the skeleton of cattle, sheep and pigs. Anatomia Histologia Embryologia 13, 222-230.

Everest, P. G. \& Scales, G. H. (1983). Pre and post weaning growth rates of ewes and lambs in the South Island. In Lamb Growth (Ed. A. S. Familton), pp. 41-46. Canterbury, New Zealand: Lincoln College.

Field, R. A., Young, O. A., Asher, G. W. \& Foote, D. M. (1985). Characteristics of male fallow deer muscle at a time of sex-related muscle growth. Growth 49, 190-201.

GalbRaith, H. \& Topps, J. H. (1981). Effect of hormones on the growth and body composition of animals. Nutrition Abstracts and Reviews - Series B 51, 521-540.

Galbraith, H., Hatendi, P. R., Alderson, E. M. \& Scaife, J. R. (1990). The effect of cimaterol and oestradiol-17 $\beta$ alone or combined on growth and body composition of wether lambs. Animal Production 51, 311-319.

Galbraith, H., Singh, S. B. \& Scaife, J. R. (1997). Response of castrated male sheep to oestrogenic and androgenic compounds implanted alone or in combination. Animal Science 64, 261-269.

GetTy, R. (Ed.) (1975). Sisson and Grossman's The Anatomy of the Domestic Animals. 5th edition. Philadelphia: Saunders.

Hammond, J. (1932). Growth and Development of Mutton Qualities in Sheep. London: Oliver and Boyd.

Hancock, D. K. L., Wagner, J. F. \& Anderson, D. B. (1991). Effects of estrogens and androgens on animal growth. In Growth Regulation in Farm Animals (Eds A. M. Pearson \& T. R. Dutson), pp. 255-297. London: Elsevier.

Hooper, A. C. B. (1978a). Muscles and bones of large and small mice compared at equal body weights. Journal of Anatomy 127, 117-123.

HoOper, A. C. B. (1978b). Bone length and muscle weight in mice subjected to genetic selection for the relative length of the tibia and radius. Life Sciences 22, 283-286.

Hubard Ocariz, J. L., Littlejohn, A. \& Robertson, I. S. (1970). A comparison of entire and ovariectomized beef heifers treated with ethylestrenol. Journal of Agricultural Science, Cambridge 74, 349-356.

Hunter, R. A., Davey, J. B. \& Buttery, P. J. (1987). Fractional rate of protein synthesis in liver and in individual muscles of lambs: effect of time of sampling following the use of the continuous infusion technique. Journal of Agricultural Science, Cambridge 108, 511-514.

Muir, L. A. (1985). Mode of action of exogenous substances on animal growth: an overview. Journal of Animal Science 61(Suppl. 2), 154-180.
PÁLsson, H. (1939). Meat qualities in the sheep with special reference to Scottish breeds and crosses. Journal of Agricultural Science, Cambridge 29, 544-626.

Peterson, A. J., Fairclough, R. J. \& Smith, J. F. (1975). Radioimmunoassay of estradiol-17 $\beta$ in bovine peripheral plasma with and without chromatography. Steroids $\mathbf{2 5}$, 487-496.

Richards, F. J. (1959). Flexible growth function for empirical use. Journal of Experimental Botany 10, 290-300.

Shanbacher, B. D. \& Ford, J. J. (1977). Gonadotropin secretion in cryptorchid and castrate rams and the acute effects of exogenous steroids treatment. Endocrinology 100, 387-393.

SHORT, R. V. (1980). The hormonal control of growth at puberty. In Growth in Animals (Ed. T. L. J. Lawrence), pp. 24 45. London: Butterworths.

Shroder, J. D. \& HANSARD, S. L. (1958). Effects of orally administered stilbestrol upon growth and upon calcium and phosphorus metabolism in lambs. Journal of Animal Science 17, 343-352.

Silberberg, M. \& Silberberg, R. (1972). Steroid hormones and bone. In The Biochemistry and Physiology of Bone, 2nd edition. Volume II (Ed. G. H. Bourne), pp. 401-484. New York: Academic Press.

Spencer, G. S. G. (1985). Hormonal systems regulating growth. A review. Livestock Production Science 12, 31-46.

SuZuKi, H. K. (1958). Effects of estradiol-17-beta-n-valerate on endosteal ossification and linear growth in the mouse femur. Endocrinology 63, 743-747.

TAN, G. Y. \& FENNESSY, P. F. (1981). The effect of castration on some muscles of red deer (Cervus elaphus L.). New Zealand Journal of Agricultural Research 24, 1-3.

TANDlER, J. \& Keller, K. (1910-1911). Uber den einfluss der Kastration auf den Organismus. IV. Die Korperform der Weiblichen Fruhkastraten des Rindes. Archives fur Entwicklungsmechanik der Organismen 31, 289-306.

Wallace, L. R. (1948). The growth of lambs before and after birth in relation to the level of nutrition. Parts I, II \& III. Journal of Agricultural Science, Cambridge 38, 93-153, 243-302, 367-401.

Wilkinson, W. S., O’Mary, C. C., Wilson, G. D., Bray, R. W., Pope, A. L. \& Casida, L. E. (1955). The effect of diethylstilbestrol upon growth, fattening, and certain carcass characteristics of full-fed and limited-fed western lambs. Journal of Animal Science 14, 866-877.

ZONDEK, B. (1936). Impairment of anterior pituitary function by follicular hormone. Lancet 231, 842-847. 